\title{
In Situ Detection of Trace Furfural in Aqueous Solution Based on Au Nanoparticle/Au Film Surface-Enhanced Raman Spectroscopy
}

\author{
Wei Qi, Weigen Chen, Fu Wan, Jingxin Zou, and Zhaoliang Gu \\ State Key Laboratory of Power Transmission Equipment \& System Security and New Technology, Chongqing University, \\ Chongqing 400044, China
}

Correspondence should be addressed to Weigen Chen; 15541342@qq.com

Received 28 October 2015; Revised 22 December 2015; Accepted 30 December 2015

Academic Editor: Haichun Liu

Copyright (C) 2016 Wei Qi et al. This is an open access article distributed under the Creative Commons Attribution License, which permits unrestricted use, distribution, and reproduction in any medium, provided the original work is properly cited.

\begin{abstract}
Furfural is an important chemical solvent and intermediate. Sensitive detection of this compound has attracted great interest in various fields. Surface-enhanced Raman spectroscopy (SERS) is a highly sensitive method for material detection because of its optical enhancement effect of plasmonic nanostructures. This study presents a simple and versatile method to synthesize a SERS substrate, where polyaminothiophenol (PATP) was used to realize the stable combination of Au nanoparticles (AuNPs) and Au film via self-assembly. The near-field electric field distribution was calculated using the finite difference time domain (FDTD) simulation to determine the parameters responsible for electric field enhancement. The simulation results show that SERS enhanced factors are sensitive to interparticle spacing and materials for solid support but insensitive to particle size. Moreover, the experimental results show that the optimized substrates with the highest Raman activity were formed by six layers of $60 \mathrm{~nm}$ AuNPs decorated on a $30 \mathrm{~nm}$ thick Au film, thereby validating the simulation results. The SERS factor of the optimal substrates is approximately 5.57 $\times 10^{3}$, and the in situ detection limit is $4.8 \mathrm{ppm}$. The 3D Raman spectra, relative standard deviation values for major peaks, and changes in signal intensity with time show the good reproducibility and stability of the substrates.
\end{abstract}

\section{Introduction}

As an important chemical solvent and intermediate, furfural is extensively utilized in industrial manufacturing, pharmaceutical field, and food production. Furfural exhibits certain toxicity and can cause adverse effects on the ecological environment and human health. For instance, furfural concentrations higher than $300 \mathrm{ppm}$ in wine can seriously damage the liver, kidney, and other organs of the body $[1,2]$. Therefore, the highly sensitive in situ detection of trace furfural in aqueous solution is of great significance. Currently, the standard detection method for furfural in water has not been established. Kang et al. [3] reported the detection limit of furfural in waste water of $143 \mathrm{ppm}$ using the high performance liquid chromatography (HPLC) method. However, the technique cannot realize highly sensitive detection and has the shortcomings of the complexity of the test procedure, long cycle period, and chromatographic column aging [4]. To overcome these limitations, surfaceenhanced Raman scattering (SERS) is applied to analyze trace furfural. This approach is less time-consuming and noncontacting because the sample is directly detected by an excited laser without separate management. SERS has great potentialities for biochemical sensing, which is due to field enhancement by factors up to 10-14 orders of magnitude [59]. The strong surface electromagnetic field enhancement of metal nanostructures is induced by localized surface plasmon resonance (LSPR) [10-12]. This phenomenon provides a novel method for detection of compounds at low concentrations.

Currently, the in situ SERS detection of aqueous furfural remains at the initial stages. Several studies $[13,14]$ experimentally and theoretically analyzed the SERS spectra of furfural collected on Ag nanoparticles, copper electrodes, and 


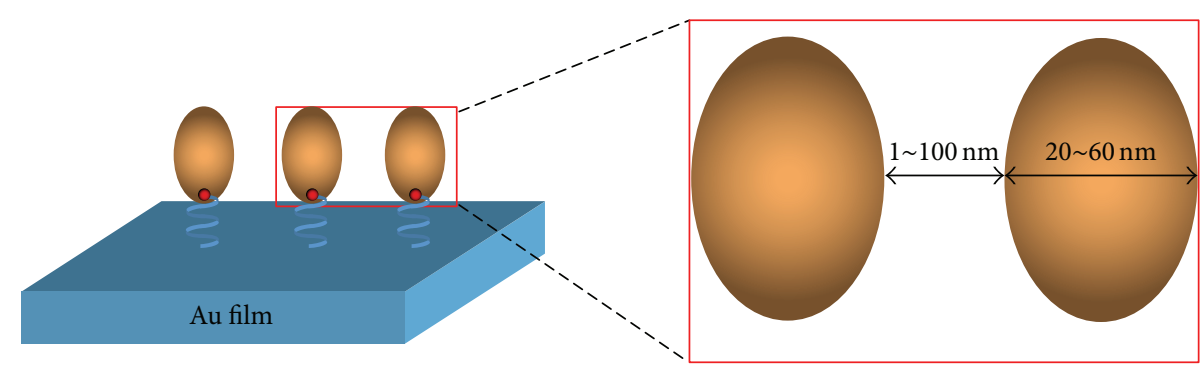

(a)
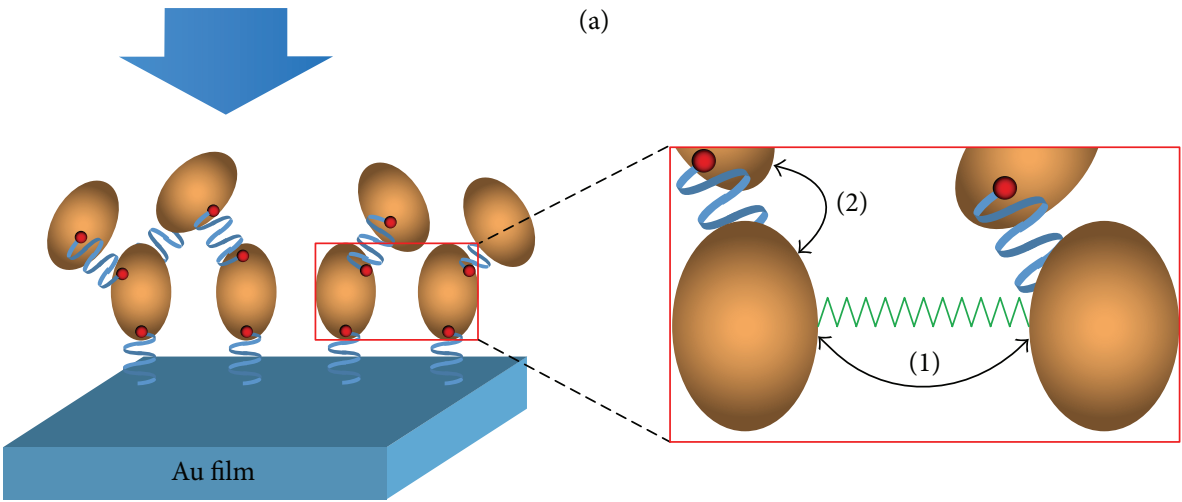

Thiol group

nM Electromagnetic coupling
(1) Hot spots between adjacent AuNPs

(2) Hot spots between AuNPs on different layers

(b)

FIGURE 1: Schematic of the basic principle of Au film decorated by (a) monolayer and (b) multiple layers of AuNPs. The interparticle spacing ranged from $1 \mathrm{~nm}$ to $100 \mathrm{~nm}$. In the Au colloid-modified Au film system, hot spots appear in the gaps between AuNPs and Au film. As the number of layers increases, additional hot spots from AuNPs on the additional layers are formed and the coupling effect is enhanced for the induced nanogap.

silver mirrors, and the corresponding adsorbed states of furfural were also discussed. However, traditional substrates can only provide finite "hot spots," where strong field enhancement occurs. It leads to SERS sensitivity of these substrates cannot meet the detection requirements. The reproducibility test, stability test of the substrates, and quantitative calculation have not been discussed. To realize highly sensitive in situ detection of trace in aqueous solution, much efforts are needed to prepare highly Raman-active substrates. Some researchers $[15,16]$ reported that a small portion of molecules absorbed at strongly enhanced locations (hot spots) can considerably contribute to the Raman signal. Moreover, the electric field enhancement exponentially increases for enlarged electromagnetic coupling with decreasing nanogaps [17]. Therefore, to obtain sufficient hot spots and strong electromagnetic coupling for preparing highly Raman-active substrates and improving detection sensitivity, we developed a strategy involving three steps: (1) rationally choosing the appropriate solid support, (2) increasing Au nanoparticles (AuNPs) density to provide sufficient hot spots and produce strong coupling effect, and (3) varying the particle size to attain tunable electromagnetic properties.

In this study, the finite difference time domain (FDTD) method was used to calculate the electric field distribution of a system containing an $\mathrm{Au}$ dimer and an $\mathrm{Au}$ film to reveal parameters affecting SERS enhancement. Based on the simulation results, a series of multiple-layered self-assembled AuNPs/Au film substrates were prepared. The effect of the assembled layers on SERS enhancement was also studied. The SERS spectra of trace furfural at different concentrations were investigated using optimal Raman-active substrates. This study proposes a novel method applicable for in situ sensitive detection of trace furfural in aqueous solutions.

\section{Structure and Preparation}

2.1. Structure of the SERS Substrate. The common synthesis procedure for SERS substrates is delineated in Figure 1. The bifunctional polyaminothiophenol (PATP) molecules were applied to bind AuNPs to the Au film surface through $\mathrm{Au}-\mathrm{S}$ covalent bonds and form uniform nanoscale arrays, which are SERS active. On this basis, additional layers of AuNPs were assembled above the existing surface. Applying bifunctional PATP of various layers can achieve different surface particle densities and nanostructures by deposited colloid particles. Compared with the Au colloid monolayer, linking multiple AuNP layers can form hot spots between AuNPs on different layers and on the same layer to produce a strong coupling effect of AuNPs for enhanced SERS performance.

2.2. Calculation of Electric Field Distribution. To gain an indepth understanding of SERS enhancement and determine 


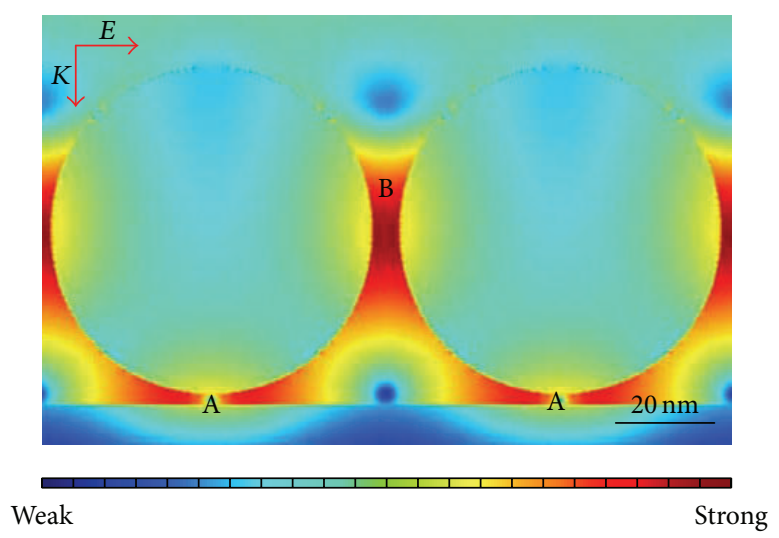

(a)

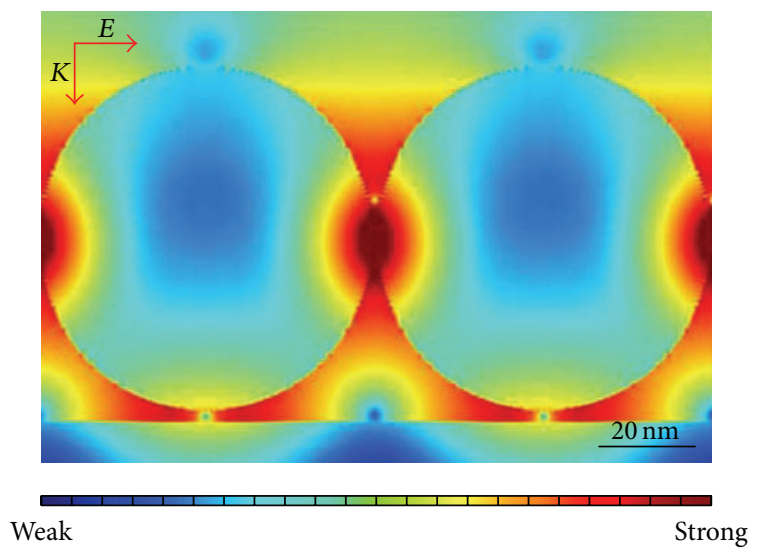

(c)

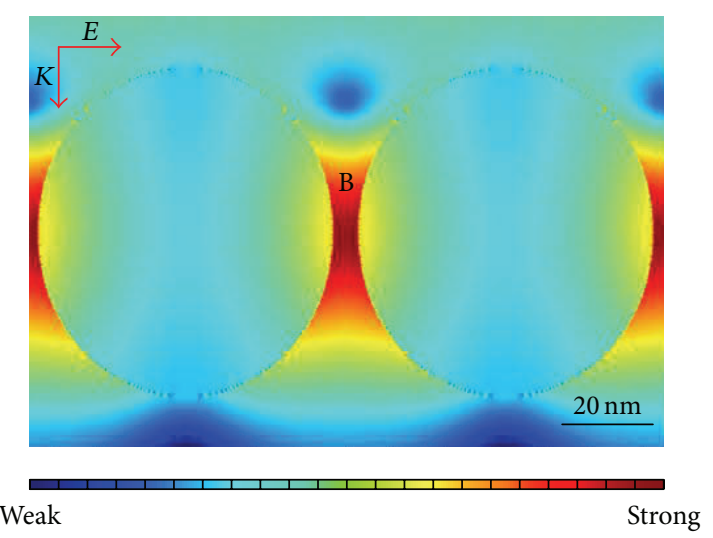

(b)

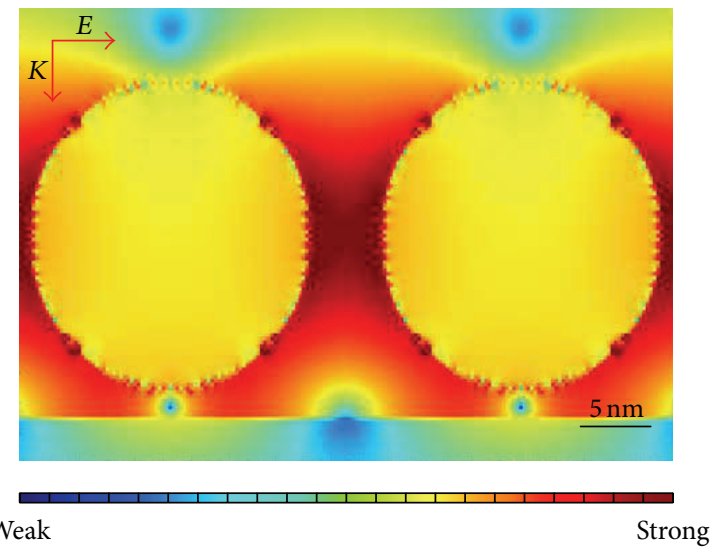

(d)

FIGURE 2: The calculated near-field electric distribution in different configurations excited at $632.8 \mathrm{~nm}$ : ((a), (b)) Au dimer (60 nm) with interparticle spacing of $5 \mathrm{~nm}$ located above (a) the Au film and (b) the $\mathrm{SiO}_{2}$ support, (c) Au dimer (60 $\mathrm{nm}$ ) with interparticle spacing of $1 \mathrm{~nm}$ located above the Au film, and (d) Au dimer $(20 \mathrm{~nm}$ ) with interparticle spacing of $5 \mathrm{~nm}$ located above the Au film. The refractive index is 1.33 for all configurations. The insets with red arrows show the propagation direction of the incident light perpendicular to the dimer structure with polarization parallel to $x$-axis.

the peak values beneficial for SERS studies, we simulated a system comprising a $30 \mathrm{~nm}$-thick Au film and two Au spheres located $2 \mathrm{~nm}$ above the film through the FDTD method to calculate the near-field enhancement. The propagation direction of the incident light is perpendicular to the dimer structure with polarization parallel to the $x$-axis, as shown by red arrows in Figure 2. All simulations used the fixed excitation wavelength $(632.8 \mathrm{~nm})$, which is close to the gold absorption band [18]. The refractive index of the ambient water medium is 1.33 . The deeper red color in the simulation results indicates higher electric field values.

To examine the influence of the support material on the near-field distribution, Figures 2(a) and 2(b) show a model I of a $60 \mathrm{~nm}$ gold dimer with $5 \mathrm{~nm}$ nanogap on a $30 \mathrm{~nm}$ thick $\mathrm{Au}$ film and $\mathrm{a} \mathrm{SiO}_{2}$ support, respectively. The peak values of $\left|E_{x}\right|^{4} /\left|E_{0}\right|^{4}$ ( $E_{0}$ is the incident electric field) in the gap between two AuNPs of $1.49 \times 10^{5}$ in Figure 2(a) are comparable with that of $1.57 \times 10^{5}$ in Figure 2(b). It is interesting to observe that the electric field in the gaps between the AuNPs and the gold support is much enhanced compared with that in the dimer-SiO${ }_{2}$ gaps. It is reported the signal from gap A is even stronger than from gap B in some cases [19]. This phenomenon indicates that the hot spots locate not only in the gaps between AuNPs, but also in the dimer-film gaps. Based on the simulation results, we preferred to use the Au film as support rather than dielectric supports $\left(\mathrm{SiO}_{2}\right)$ to provide more hot spots.

To examine the influence of interparticle distance and particle radius on the scattered field, we performed similar calculations for additional model II at the fixed distance $(2 \mathrm{~nm})$ above the Au film ( $30 \mathrm{~nm}$ thickness). The calculated electric field enhancement values in Figures 2(c) and 2(d) are $1.17 \times 10^{9}\left(60 \mathrm{~nm}\right.$ size, $1 \mathrm{~nm}$ gap) and $1.82 \times 10^{6}(20 \mathrm{~nm}$ size, $5 \mathrm{~nm}$ gap), respectively. For all configurations, the highest electric field was observed in the dimers of Figure 2(c) which is three or four orders of magnitude higher than that in other cases. These dimers show stronger electromagnetic coupling at shorter interparticle distance. The results agree with the previous conclusion that the SERS EFs are higher because of the nonlinear increase of the local electric field when the gap 
between the particles is less than $10 \mathrm{~nm}$ [17]. It is investigated that the proposed system is more sensitive to the distance between two AuNPs than particle size.

Ideally, numerous hot spots could be formed and higher electric field values could appear on optimized nanostructures including nanogap, particle radius, and support material which contribute to greater enhancement of the SERS signal of trace furfural.

2.3. Preparation of the SERS Substrate. All glassware was washed with "Piranha solution" (1:3 mixture of $30 \% \mathrm{H}_{2} \mathrm{O}_{2}$ and concentrated $\mathrm{H}_{2} \mathrm{SO}_{4}$ ) at $90^{\circ} \mathrm{C}$ for approximately $1 \mathrm{~h}$. The $\mathrm{Au}$ colloids were prepared by citrate reduction as previously reported [20]. All samples were stored in a sealed vial at $4^{\circ} \mathrm{C}$. After thorough washing with a copious amount of $\mathrm{H}_{2} \mathrm{O}$ and ethanol, the $\mathrm{Au}$ film was immersed in $0.1 \mathrm{~mol} / \mathrm{L}$ PATP in ethanol for $12 \mathrm{~h}$. After copious rinsing, all substrates were immersed into the solution of $\mathrm{Au}$ colloids for $12 \mathrm{~h}$. The immersion of substrates in Au colloids was repeated for several times to produce multiple-layered self-assembled substrates, which can increase the density of Au particle.

\section{Raman Experiment}

All Raman spectra were measured excited by a laser at $632.8 \mathrm{~nm}$ (the spot area is approximately $5 \mu \mathrm{m}^{2}$ ). The carbonation of substrates could occur and furfural is easily decomposed under high excitation power. Thus, the integration time was set as $1 \mathrm{~s}$, and the power was $50 \mu \mathrm{W}$. The Raman spectra were collected in backscattering geometry through a microscope objective (magnitude $\times 100$, numerical aperture 0.9). A Raman spectrometer (SR-500I-C) has three pieces of grating: 1800, 1200, and 600 grooves/mm. CCD (Andor DU416A-LDC-DD) was cooled with liquid nitrogen (fully depleted back illuminated type, $2000 \times 256$ pixels, quantum efficiency $>90 \%$, dark noise $\leq 0.0006$ electron/s/pixel). The molecular probe is furfural at various concentrations.

3.1. Optimization of Self-Assembled Layers. To confirm the effect of the number of AuNPs layers on the SERS enhancement, a set of self-assembled AuNPs (average diameter $62.33 \mathrm{~nm}$, standard deviation $6.39 \mathrm{~nm}$ )/Au film (30 nm thickness) were prepared. The SEM photos of the samples assembled from one to six layers are shown in Figure 3. Figure 3(a) presents the SEM image of the gold colloidal monolayers, which are uniformly distributed on the same plane. The configuration of two layers appears in Figure 3(b) indicating the successful fabrication of the second layer AuNPs after chemically modifying the Au film with ordered monolayer of PATP. Most AuNPs exist in isolation rather than immobilized aggregation. Prolonged immersion could not produce morphological changes which gives a proof of nanoparticle saturation. The adsorption of PATP cannot influence the existing AuNP distribution because of the strong adhesion of thiol groups to noble metal. As the number of assembled layers increases, the density of the surface AuNPs greatly increases, and abundant particle clusters simultaneously appear (Figures 3(c)-3(f)). This phenomenon can be explained by the fact that single AuNP of additional layer may locate above the existing single nanoparticle or bridging above the adjacent nanoparticles, and multiple particles may be simultaneously adsorbed on a single particle.

The SERS spectra collected on six-layered self-assembled substrates in deionized water are shown in Figure 4(a). High background peaks at around 1075, 1141, 1185, 1334, 1387, 1432,1576 , and $1616 \mathrm{~cm}^{-1}$ were observed, which agrees with previous findings $[21,22]$. The SERS spectra of $0.1 \mathrm{~mol} / \mathrm{L}$ furfural aqueous solution collected on a set of Au-decorated substrates with different numbers of layers were investigated, shown in Figure 4(b). It was investigated that the SERS peaks from the substrates and furfural molecules overlap seriously, which greatly interferes with the SERS detection of trace furfural. However, the SERS peaks of furfural at 1478 and $1665 \mathrm{~cm}^{-1}$ remain visible excluding background peaks. The furfural stretching vibration peak $\left(1665 \mathrm{~cm}^{-1}\right)$ was extracted and chosen as SERS characteristic peak because of its strong intensity and lack of an overlapping section with the other peaks. Moreover, the results show that the signals collected on Au film substrate without AuNPs slightly increase because the substrates provide extremely limited hot spots. It is reported that the enhancement factors for Raman intensity include nanoparticle spacing and the number of hot spots $[17,18]$. With other experimental parameters fixed, the SERS intensity of the characteristic peak at $1665 \mathrm{~cm}^{-1}$ becomes stronger with the increased assembled layers. This phenomenon reveals the formation of numerous hot spots and the decrease in nanogaps, which is in good agreement with the previous simulation results shown in Figures 2(a) and 2(c).

3.2. Calculation of the SERS Enhancement Factor. To further evaluate the SERS enhancement effect, SERS enhancement factor (EF) has been calculated. The SERS intensities of $0.1 \mathrm{~mol} / \mathrm{L}$ furfural at $1665 \mathrm{~cm}^{-1}$ on the substrates assembled with different layers (from six layers to one layer) are 1250, $790,586,289,258$, and 187 counts, respectively, whereas the normal Raman scattering (NRS) spectrum under the same conditions is approximately 100 counts (Figure 4(b)). Hence, the signal intensity of the six-layered substrate is approximately seven times higher than that of the monolayer. Due to the in situ detection condition without drying process, SERS EFs are defined according to literature [23]:

$$
E=\frac{I_{\text {SERS }} / N_{\text {SERS }}}{I_{\text {solu }} / N_{\text {solu }}},
$$

where $I_{\text {SERS }}$ and $I_{\text {solu }}$ are the SERS peak intensities of furfural adsorbed on the substrate surface and the NRS peak intensity of furfural in aqueous solution, respectively; $N_{\text {SERS }}$ and $N_{\text {solu }}$ are the number of furfural molecules on substrate surface and in aqueous solution within the laser spot area, respectively.

From the SEM photos, AuNPs are irregularly distributed on the $\mathrm{Au}$ film. It is difficult to accurately estimate $N_{\text {SERS }}$ due to the difficulties in defining the effective surface area of AuNPs. To simplify the estimation of $N_{\text {SERS }}$, we calculate the average number of AuNPs on the one-layered substrates in $1 \mu \mathrm{m}^{2}$. Thus, the total surface area of the one-layered substrates is calculated to be around $2.7 \times 10^{5} \mathrm{~nm}^{2}$. If we 


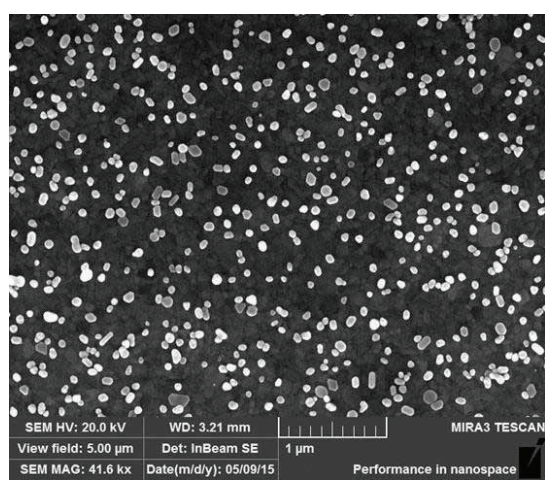

(a)

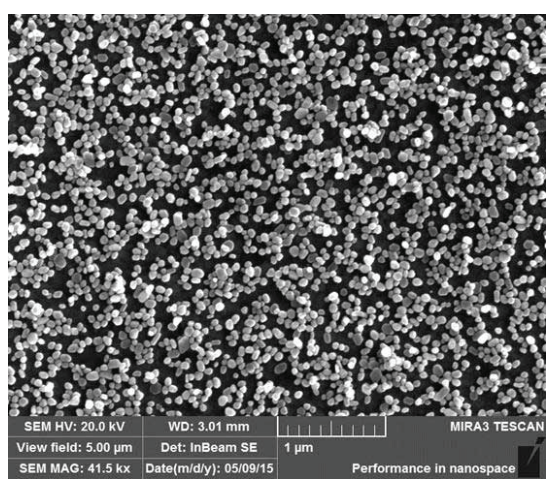

(d)

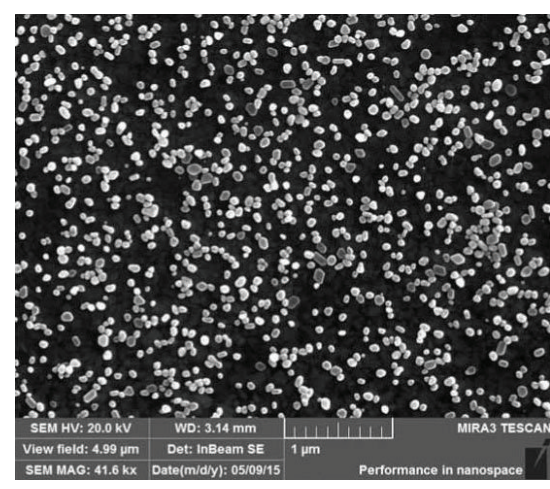

(b)

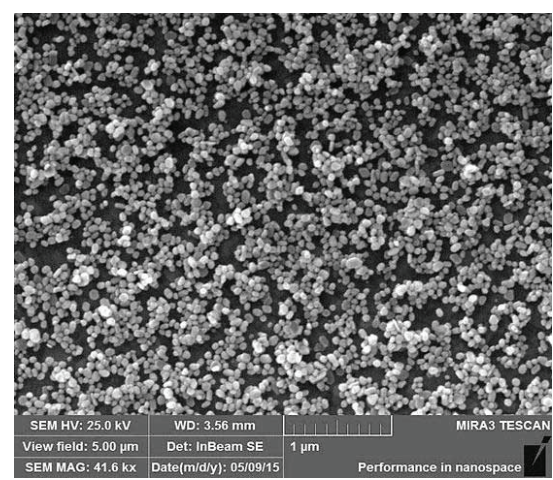

(e)

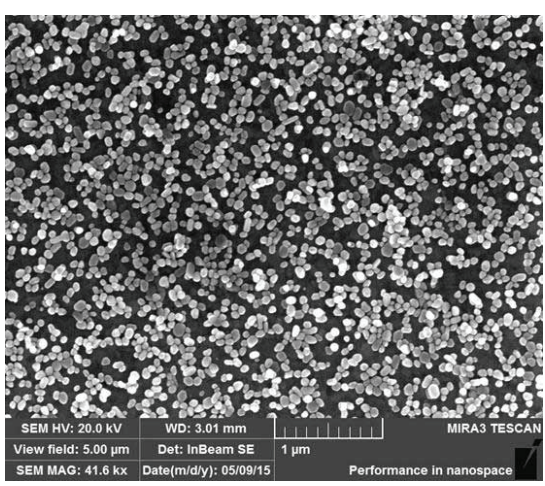

(c)

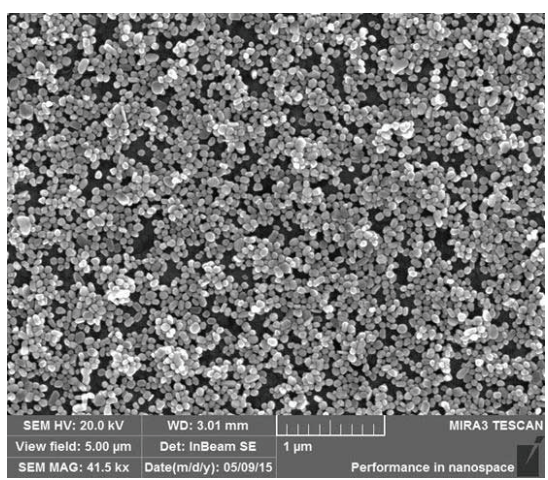

(f)

Figure 3: SEM images of a set of Au-decorated substrates assembled from monolayer to six layers.

TABLE 1: SERS EFs for major Raman peaks for different SERS samples.

\begin{tabular}{lcccccc}
\hline \multirow{2}{*}{ Raman peak $\left(\mathrm{cm}^{-1}\right)$} & \multicolumn{3}{c}{ The number of assembled layers } \\
& 1 layer & 2 layers & 3 layers & 4 layers & 5 layers & 6 layers \\
\hline $1665 \mathrm{~cm}^{-1}$ & $2.07 \times 10^{3}$ & $2.38 \times 10^{3}$ & $2.22 \times 10^{3}$ & $3.76 \times 10^{3}$ & $4.22 \times 10^{3}$ & $5.57 \times 10^{3}$ \\
$1478 \mathrm{~cm}^{-1}$ & $6.05 \times 10^{2}$ & $6.33 \times 10^{2}$ & $5.81 \times 10^{2}$ & $8.05 \times 10^{2}$ & $6.98 \times 10^{2}$ & $1.24 \times 10^{3}$ \\
\hline
\end{tabular}

assume that a single layer of furfural molecules is adsorbed on the AuNPs surface and furfural molecule area is around $5 \mathrm{~nm}^{2}$ [24], $N_{\text {SERS }}$ is estimated to be around $5.4 \times 10^{4}$ per $1 \mu \mathrm{m}^{2}$. Considering the SEM results, we further assume the number of AuNPs increased by around 20\% per increased single layer. $N_{\text {SERS }}$ on different SERS substrates can be calculated. $N_{\text {solu }}$ can be expressed as a function of the laser spot radius $a$, the depth by which the laser passes through the aqueous solution $h$ and the analyte concentration $M$ :

$$
N_{\text {solu }}=N_{A} \cdot \pi a^{2} \cdot h \cdot M
$$

The parameter values in formula are $A_{1}$ of $1 \mu \mathrm{m}^{2}, h$ of $1 \mathrm{~mm}$, Avogadro constant $N_{A}$ of $6.02 \times 10^{23}$, and $M$ of $0.1 \mathrm{~mol} / \mathrm{L}$. With these values, we can calculate the SERS EF values for furfural on different SERS substrates at 1478 and $1665 \mathrm{~cm}^{-1}$, given in Table 1 . The EF values increase with increasing number of assembled layers. Theoretically, interparticle spacing could partially decrease to $1-2 \mathrm{~nm}$ with increased assembled layers because the bifunctional molecules PATP used to covalently bind the additional AuNPs are approximately 1-2 nm [25]. There is a report [26] stating that when AuNPs on surface are close-packed and aggregate approximating a bulk surface, most of the SERS effect is lost. Therefore, we speculate that the AuNPs/Au film substrates could be weakly enhanced when the layers continuously increase to a certain extent. The maximum EF value of about $5.57 \times 10^{3}$ is observed in the six-layered selfassembled substrates. Hence, the experimental SERS EF value is up to 6 orders of magnitude lower than the simulated SERS $\mathrm{EF}$ value. This phenomenon can be explained by the fact that the simulation results are the maximum EF values of one point in the near field with fixed nanogaps, whereas the experimental results are the average SERS EF values in the far field with unavoidable inhomogeneous nanogaps. Moreover, the SERS EF values at $1478 \mathrm{~cm}^{-1}$ and $1665 \mathrm{~cm}^{-1}$ are different. This can be understood by the fact that the incident light wavelength and scattering wavelength are unlikely to meet all the resonance conditions of the scattered light for the finite plasmon resonance band [27]. 


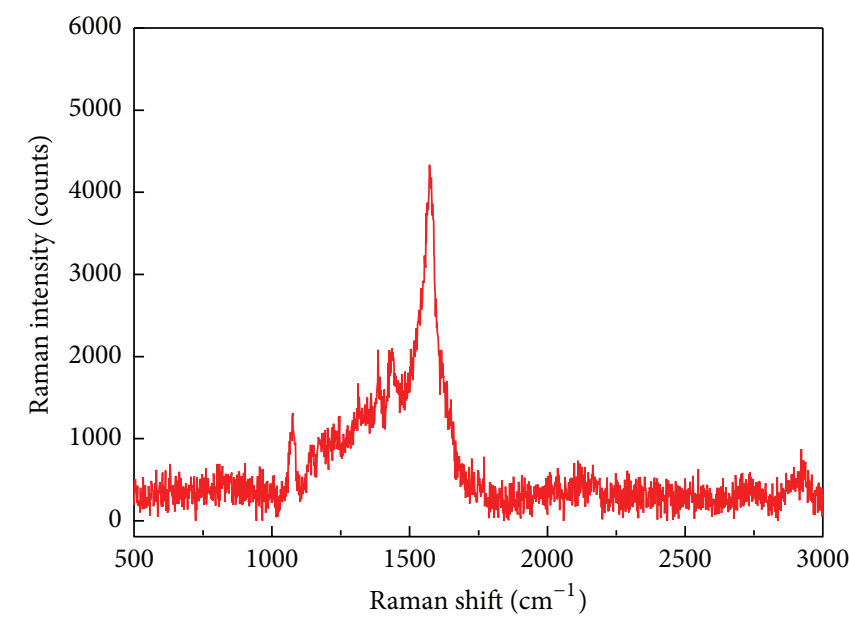

(a)

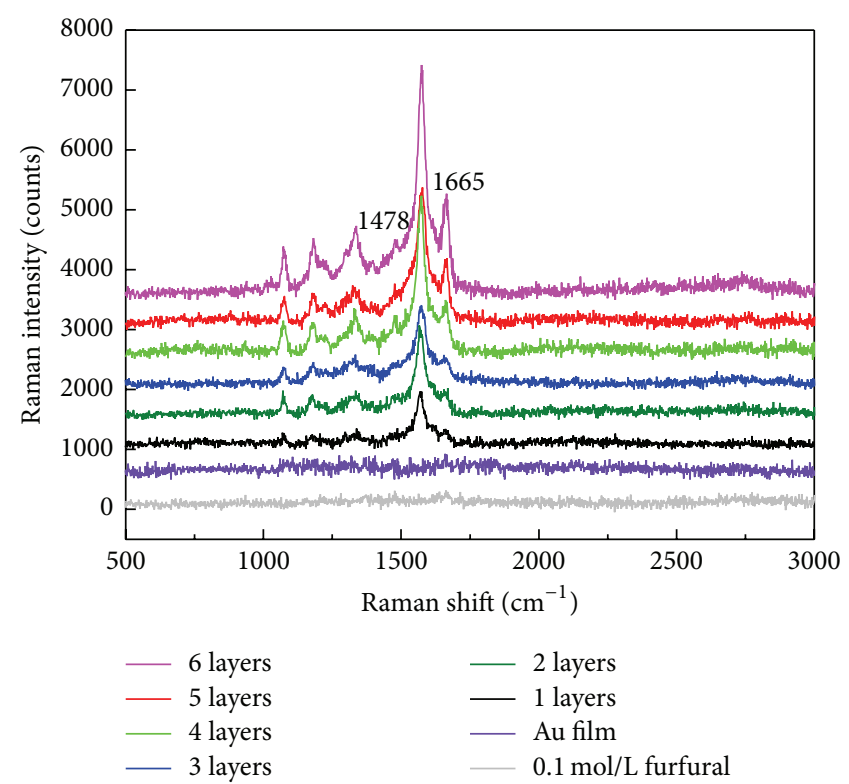

(b)

FIGURE 4: SERS spectra of (a) the SERS substrate sample (six-layered) and (b) $0.1 \mathrm{~mol} / \mathrm{L}$ furfural aqueous solution collected on different substrate samples assembled from one to six layers and Au film without AuNPs. The bottom line (grey trace) indicates the NRS signal of $0.1 \mathrm{~mol} / \mathrm{L}$ furfural.

To gain better SERS enhancement performance, the optimization for our substrate could be (1) constituting sufficient hot spots to further enhance the local electromagnetic field between adjacent AuNPs, (2) decreasing the nanogaps to enhance electromagnetic coupling, (3) optimizing specific test procedures, such as immersion time in Au sol and PATP in ethanol, and (4) protecting the $\mathrm{Au}$ from oxidation to improve the stability.

3.3. Calculation of the Detection Limit. To investigate the SERS sensitivity of the optimal six-layered self-assembled substrates, SERS spectra of furfural at different concentrations were collected (Figure 5(a)). The SERS signal of the characteristic peak at $1665 \mathrm{~cm}^{-1}$ decreases with decreasing furfural concentration. When the concentration of furfural is less than $10^{-3} \mathrm{~mol} / \mathrm{L}$, portions of the SERS peaks from the substrates $\left(1075,1185,1334\right.$, and $\left.1576 \mathrm{~cm}^{-1}\right)$ become weaker because the laser power is slightly decreased to avoid the decomposition of low-concentration adsorbed furfural molecules. To directly display the statistical distribution of the characteristic peak at $1665 \mathrm{~cm}^{-1}$, we plotted a histogram, where the intensity and its standard deviation value on the $y$-axis correspond to the number of decorated layers (Figure 5(b)). Our experimental results show that the SERS signal of furfural $\left(10^{-4} \mathrm{~mol} / \mathrm{L}\right)$ at $1665 \mathrm{~cm}^{-1}$ is still distinguishable from the background signal. The integration time is $1 \mathrm{~s}$, the intensity of characteristic peak at $1665 \mathrm{~cm}^{-1}$ is 183 counts, and the baseline noise (baseline standard deviation) is 32 counts. Hence the signal-to-noise $(\mathrm{S} / \mathrm{N})$ ratio is approximately 6 , which indicates that this novel method can realize the in situ detection of $4.8 \mathrm{ppm}$ furfural in aqueous solution.
3.4. Reproducibility and Stability of the Substrates. The reproducibility of the substrate was tested by Raman activity of randomly selected 6 points, and we choose six-layered decorated substrates as representative. The 3D Raman spectrum provides a simple and intuitive method to assess signal reproducibility. Figure 6(a) shows the 3D SERS spectra of $10^{-2} \mathrm{~mol} / \mathrm{L}$ furfural molecules on 6 different spots with different colors. It is apparent to see that the six-layered substrate has high reproducibility. To further estimate signal reproducibility, the relative standard deviation (RSD) is commonly used. The RSD value of the signal intensity of the characteristic peak at $1665 \mathrm{~cm}^{-1}$ is $5.08 \%$, which meets the requirements (less than 20\%) proposed by Natan [28]. The as-prepared SERS substrates have potentiality for furfural detection because of their good consistency and reproducibility.

To test the stability of six-layered substrates selected as representative, we prepared the substrates to detect the changes in the SERS intensity of $10^{-2} \mathrm{~mol} / \mathrm{L}$ furfural with time. After different days' aerobic exposure in air, we plotted the SERS intensity and its standard deviation value versus exposure time, shown in Figure 6(b). Qualitatively, the signal intensity decreases to $72.5 \%$ after 14 days' aerobic exposure. Then it is seen that they still have obvious reduction in 28 days, whereas a plateau appeared after 28 days' aerobic exposure. The experimental results show that the substrate has good stability. It is speculated that because gold nanoparticles stack, the oxidized AuNPs on the surface could provide protective effect for the underlying AuNPs. Therefore, the underlying AuNPs are still Raman active even if the surface AuNPs oxidize seriously. 


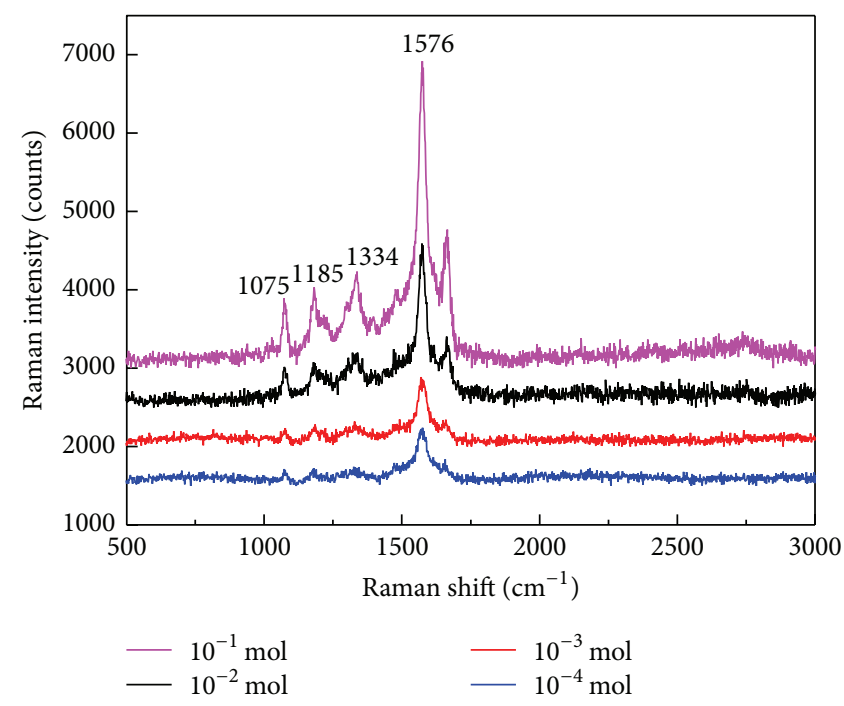

(a)

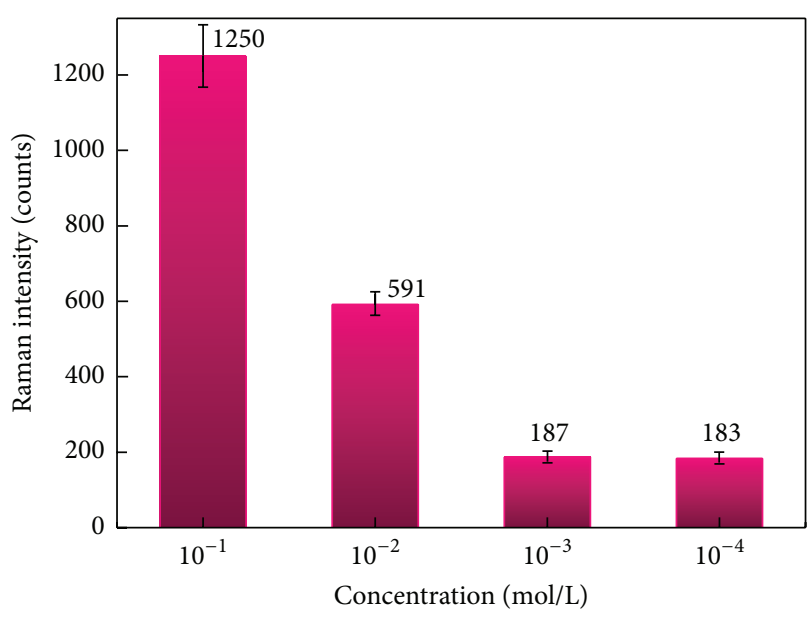

(b)

FIGURE 5: (a) SERS signals of furfural at different concentrations collected on the six-layered substrates and (b) the SERS intensity of furfural peak $\left(1665 \mathrm{~cm}^{-1}\right)$ at different concentration.

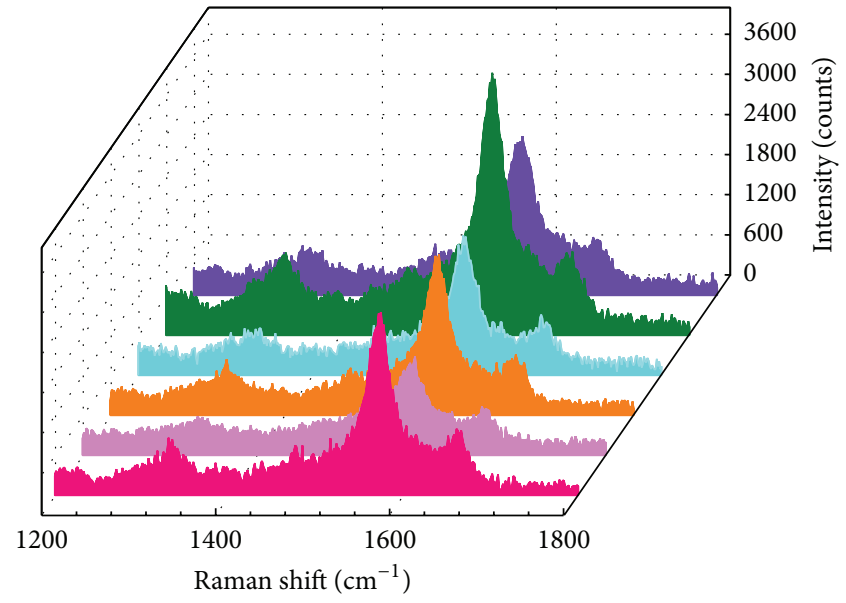

(a)

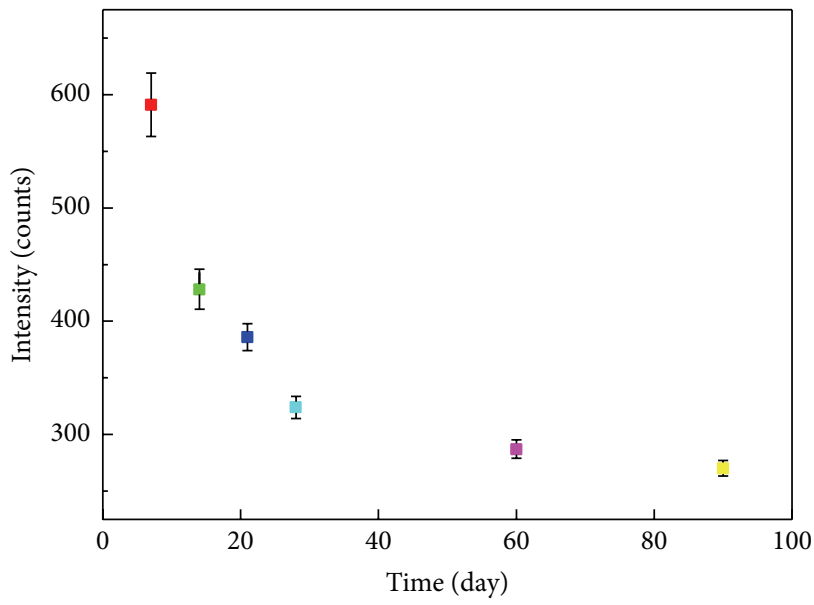

(b)

FIGURE 6: (a) The SERS spectra of $10^{-2} \mathrm{~mol} / \mathrm{L}$ furfural solution at six randomly selected points collected on six-layered substrates. (b) Changes in the SERS intensity of furfural characteristic peak $\left(1665 \mathrm{~cm}^{-1}\right)$ with time.

\section{Conclusion}

In this study, a model composed of an $\mathrm{Au}$ dimer and an $\mathrm{Au}$ film was established to calculate the near-field distribution of metallic nanostructure via FDTD method to simulate the experimental system. It is concluded that SERS enhancement mainly depends on the distance between adjacent AuNPs and conductive support. On the basis, we demonstrated a type of SERS-based biosensing arrays for in situ detection of trace furfural in aqueous solution. We investigated the influence of the number of assembled layers on the enhancement performance. The optimal SERS-active substrates are the six-layered substrates with the SERS EF value of $5.57 \times 10^{3}$, which can realize the SERS detection of furfural at concentration below $4.8 \mathrm{ppm}$. The 3D Raman spectra, RSD values of the furfural characteristic peak, and changes in the SERS intensity with time indicate that the SERS-active AuNPs/Au film substrate samples exhibit improved reproducibility and stability.

\section{Conflict of Interests}

The authors declare that they have no financial or personal relationship with any people or any organization that may inappropriately influence their work, and there is no professional or commercial interest of any kind in all of the commercial entities mentioned in their paper. 


\section{Acknowledgments}

Thanks are due to Funds for Innovative Research Groups of China (51321063, 51021005) and Special Funds for the Development of National Major Scientific Instruments and Equipment (2012YQ160007).

\section{References}

[1] M. J. Taherzadeh, R. Eklund, L. Gustafsson, C. Niklasson, and G. Lidén, "Characterization and fermentation of dilute-acid hydrolyzates from Wood," Industrial and Engineering Chemistry Research, vol. 36, no. 11, pp. 4659-4665, 1997.

[2] E. Palmqvist and B. Hahn-Hägerdal, "Fermentation of lignocellulosic hydrolysates. I: inhibition and detoxification," Bioresource Technology, vol. 74, no. 1, pp. 17-24, 2000.

[3] C. Kang, X. Tang, H. Yu, and X. Lin, "Determination of furfural content in furfural wastewater by highly efficient liquid chromatography," Industrial Water Treatment, vol. 27, no. 6, pp. 67-68, 2007.

[4] L. Zi-qiang, Z. Yong, and L. Yu-hai, "Study of high performance liquid chromatography detecting furfural content in transformer oil," Qinghai Electric Power, vol. 28, no. 2, pp. 1-5, 2009.

[5] G. Wang, H.-Y. Park, R. J. Lipert, and M. D. Porter, "Mixed monolayers on gold nanoparticle labels for multiplexed surfaceenhanced Raman scattering based immunoassays," Analytical Chemistry, vol. 81, no. 23, pp. 9643-9650, 2009.

[6] J.-H. Kim, J.-S. Kim, H. Choi et al., "Nanoparticle probes with surface enhanced Raman spectroscopic tags for cellular cancer targeting," Analytical Chemistry, vol. 78, no. 19, pp. 6967-6973, 2006.

[7] H.-Y. Wu, C. J. Choi, and B. T. Cunningham, "Plasmonic nanogap-enhanced Raman scattering using a resonant nanodome array," Small, vol. 8, no. 18, pp. 2878-2885, 2012.

[8] H.-C. Lo, H.-I. Hsiung, S. Chattopadhyay et al., "Label free subpicomole level DNA detection with Ag nanoparticle decorated $\mathrm{Au}$ nanotip arrays as surface enhanced Raman spectroscopy platform," Biosensors and Bioelectronics, vol. 26, no. 5, pp. 24132418, 2011.

[9] Y. S. Huh and D. Erickson, "Aptamer based surface enhanced Raman scattering detection of vasopressin using multi-layer nanotube arrays," Biosensors and Bioelectronics, vol. 25, no. 5, pp. 1240-1243, 2010.

[10] T. Itoh, K. Yoshida, V. Biju, Y. Kikkawa, M. Ishikawa, and Y. Ozaki, "Second enhancement in surface-enhanced resonance Raman scattering revealed by an analysis of anti-Stokes and Stokes Raman spectra," Physical Review B-Condensed Matter and Materials Physics, vol. 76, no. 8, Article ID 085405, 2007.

[11] M. Moskovits, "Surface-enhanced Raman spectroscopy: a brief retrospective," Journal of Raman Spectroscopy, vol. 36, no. 6-7, pp. 485-496, 2005.

[12] P. L. Stiles, J. A. Dieringer, and N. C. Shah, "Surface-enhanced Raman spectroscopy," Annual Review of Analytical Chemistry, vol. 1, no. 17, pp. 601-626, 2008.

[13] T. J. Jia, B. Han, and X.-M. Li, "Vibrational modes study of furfural using SERS-measurement and DFT method," Journal of Zhongzhou University, vol. 27, no. 1, pp. 114-116, 2010.

[14] Q. H. Zhang, "A study of SERS spectra of furfural molecule absorbed on the surface of copper electrode," Journal of Shangqiu Vocational \& Technical College, vol. 9, no. 2, pp. 6062, 2010.
[15] E. C. Le Ru, P. G. Etchegoin, and M. Meyer, "Enhancement factor distribution around a single surface-enhanced Raman scattering hot spot and its relation to single molecule detection," The Journal of Chemical Physics, vol. 125, no. 20, Article ID 204701, 2006.

[16] C. A. Goss, D. H. Charych, and M. Majda, "Application of (3-mercaptopropyl)trimethoxysilane as a molecular adhesive in the fabrication of vapor-deposited gold electrodes on glass substrates," Analytical Chemistry, vol. 63, no. 1, pp. 85-88, 1991.

[17] D. Radziuk and H. Möhwald, "Surpassingly competitive electromagnetic field enhancement at the silica/silver interface for selective intracellular surface enhanced Raman scattering detection," ACS Nano, vol. 9, no. 3, pp. 2820-2835, 2015.

[18] X. Wang, M. Li, L. Meng et al., "Probing the location of hot spots by surface-enhanced Raman spectroscopy: toward uniform substrates," ACS Nano, vol. 8, no. 1, pp. 528-536, 2014.

[19] Y. Fang and Y. Huang, "Electromagnetic field redistribution in hybridized plasmonic particle-film system," Applied Physics Letters, vol. 102, no. 15, Article ID 153108, 8 pages, 2013.

[20] G. Frens, "Controlled nucleation for the regulation of the particle size in monodisperse gold suspensions," Nature, vol. 241, no. 105, pp. 20-22, 1973.

[21] M. Sun, Y. Huang, and L. Xia, “The $\mathrm{pH}$-controlled plasmonassisted surface photocatalysis reaction of 4-aminothiophenol to p,p'-dimercaptoazobenzene on $\mathrm{Au}, \mathrm{Ag}$, and Cu colloids," Journal of Physical Chemistry C, vol. 115, no. 19, pp. 9629-9636, 2011.

[22] M. Osawa, N. Matsuda, and K. Yoshii, "Charge transfer resonance Raman process in surface-enhanced Raman scattering from p-aminothiophenol adsorbed on silver: Herzberg-Teller contribution," Journal of Physical Chemistry, vol. 98, no. 48, pp. 12702-12707, 1994.

[23] B. Ren, G.-K. Liu, X.-B. Lian, Z.-L. Yang, and Z.-Q. Tian, "Raman spectroscopy on transition metals," Analytical \& Bioanalytical Chemistry, vol. 388, no. 1, pp. 29-45, 2007.

[24] T. J. Jia, The Elementary Study on the Chinese Famous Liquor by Spectral Method, Henan University, 2007.

[25] K. C. Grabar, P. C. Smith, M. D. Musick et al., "Kinetic control of interparticle spacing in Au colloid-based surfaces: rational nanometer-scale architecture," Journal of the American Chemical Society, vol. 118, no. 5, pp. 1148-1153, 1996.

[26] K. C. Grabar, R. Griffith Freeman, and M. B. Hommer, "Preparation and characterization of Au colloid monolayers," Analytical Chemistry, vol. 67, no. 4, pp. 735-743, 1995.

[27] E. C. Le Ru, E. Blackie, M. Meyer, and P. G. Etchegoint, "Surface enhanced Raman scattering enhancement factors: a comprehensive study," The Journal of Physical Chemistry C, vol. 111, no. 37, pp. 13794-13803, 2007.

[28] M. J. Natan, "Concluding remarks: surface enhanced Raman scattering," Faraday Discussions, vol. 132, pp. 321-328, 2006. 

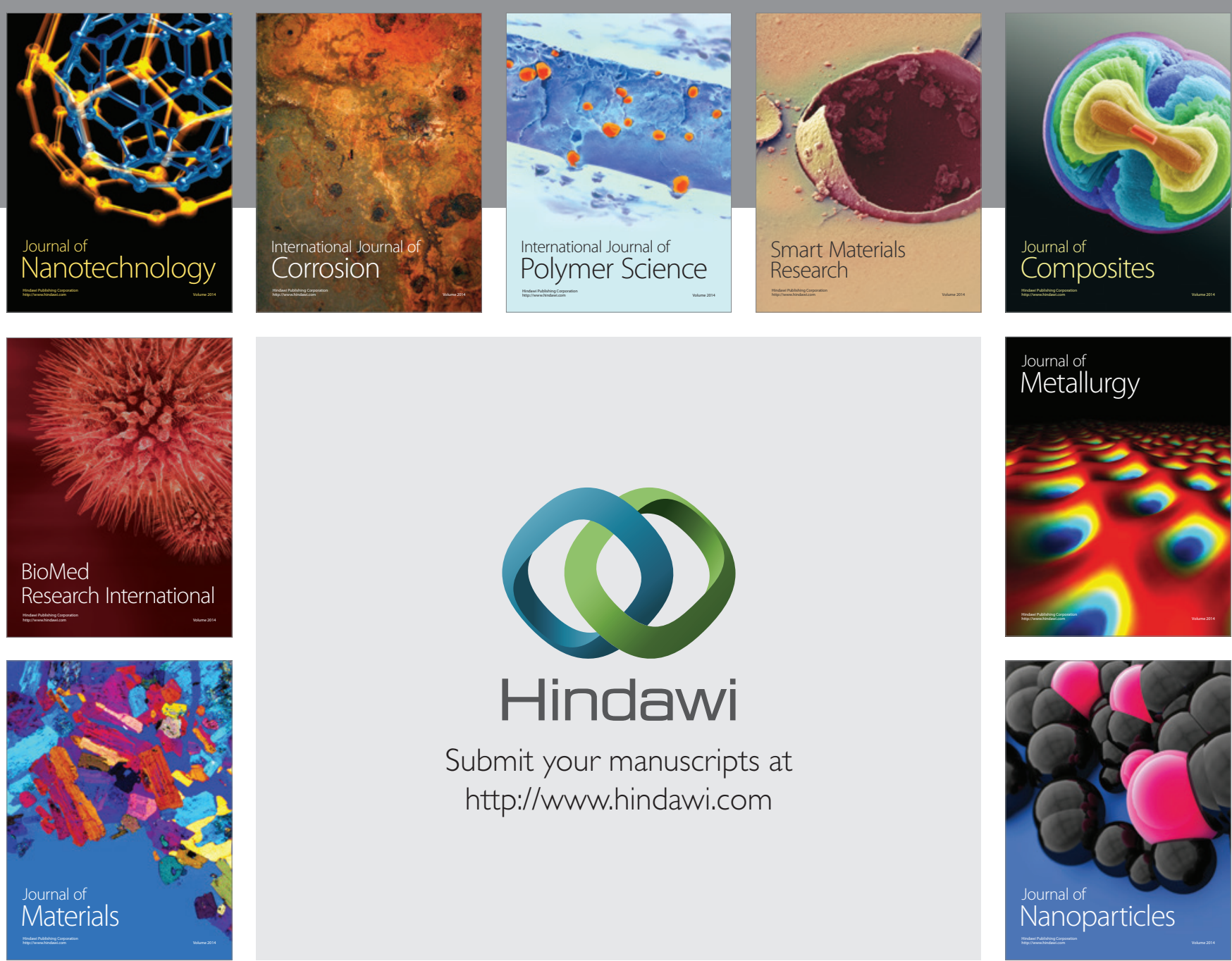

\section{Hindawi}

Submit your manuscripts at

http://www.hindawi.com

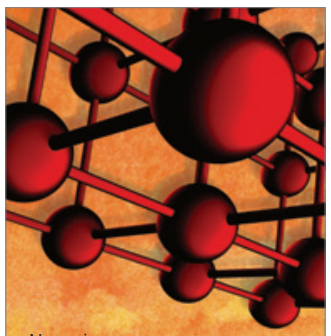

Materials Science and Engineering
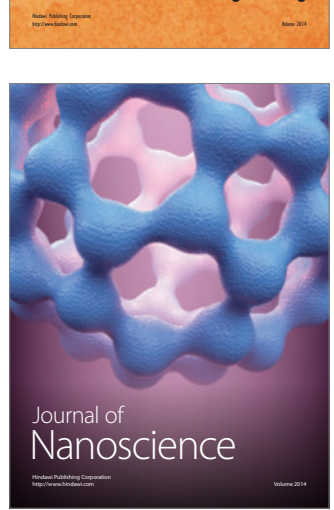
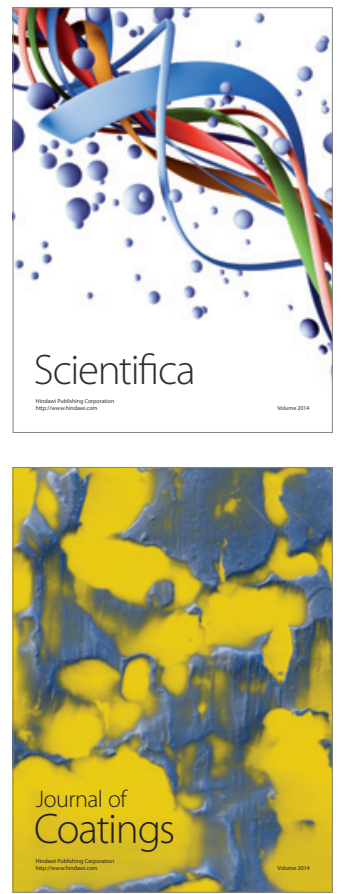
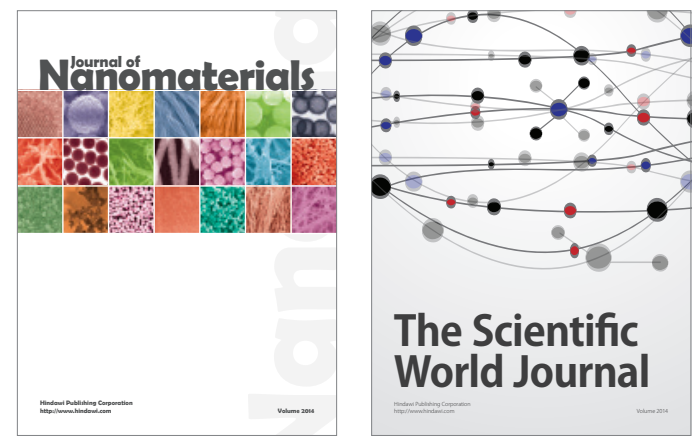

The Scientific World Journal
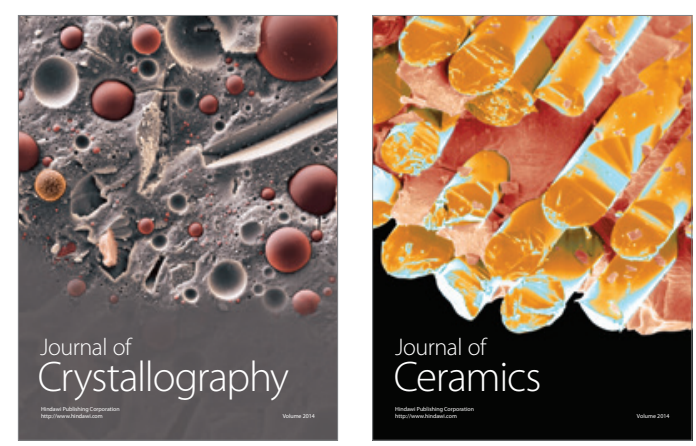
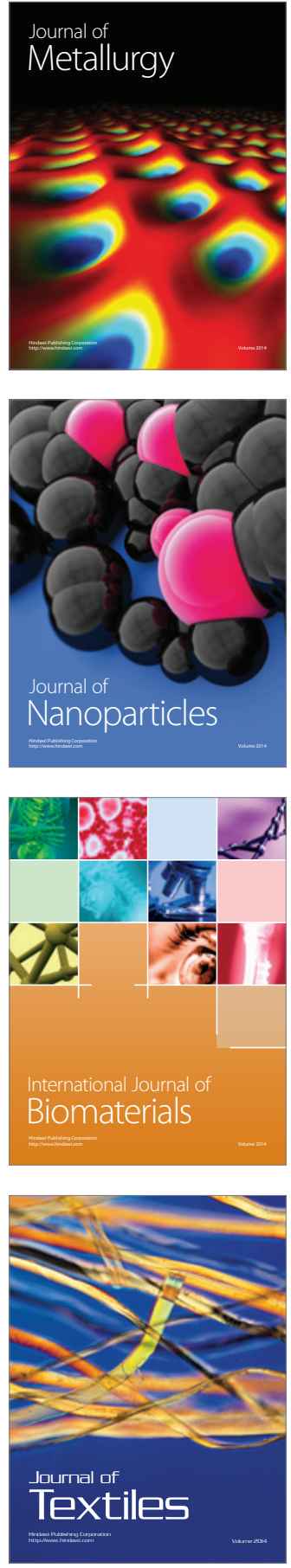